\title{
Automated Breast Ultrasound: Technical Aspects, Impact on Breast Screening, and Future Perspectives
}

\author{
Iris Allajbeu ${ }^{1,2}$ (D) Sarah E Hickman ${ }^{1} \cdot$ Nicholas Payne $^{1} \cdot$ Penelope Moyle $^{2} \cdot$ Kathryn Taylor $^{2} \cdot$ Nisha Sharma $^{3}$. \\ Fiona J Gilbert ${ }^{1,2}$
}

Accepted: 2 June 2021 / Published online: 23 June 2021

(C) The Author(s) 2021

\begin{abstract}
Purpose of Review Automated breast ultrasound (ABUS) is a three-dimensional imaging technique, used as a supplemental screening tool in women with dense breasts. This review considers the technical aspects, pitfalls, and the use of ABUS in screening and clinical practice, together with new developments and future perspectives.

Recent Findings ABUS has been approved in the USA and Europe as a screening tool for asymptomatic women with dense breasts in addition to mammography. Supplemental US screening has high sensitivity for cancer detection, especially early-stage invasive cancers, and reduces the frequency of interval cancers. ABUS has similar diagnostic performance to handheld ultrasound (HHUS) and is designed to overcome the drawbacks of operator dependence and poor reproducibility. Concerns with ABUS, like HHUS, include relatively high recall rates and lengthy reading time when compared to mammography. ABUS is a new technique with unique features; therefore, adequate training is required to improve detection and reduce false positives. Computer-aided detection may reduce reading times and improve cancer detection. Other potential applications of ABUS include local staging, treatment response evaluation, breast density assessment, and integration of radiomics.

Summary ABUS provides an efficient, reproducible, and comprehensive supplemental imaging technique in breast screening. Developments with computer-aided detection may improve the sensitivity and specificity as well as radiologist confidence and reduce reading times, making this modality acceptable in large volume screening centers.
\end{abstract}

Keywords Automated breast ultrasound $\cdot$ Artifacts $\cdot$ Screening $\cdot$ Dense breasts

\section{Introduction}

Mammography is the primary modality used for breast cancer screening, with an estimated reduction in mortality of $20 \%$ [1]. Mammographic sensitivity drops to $61 \%$ in women with

This article is part of the Topical Collection on Breast Cancer Imaging and Screening

Iris Allajbeu

ia359@cam.ac.uk

1 Department of Radiology, University of Cambridge School of Clinical Medicine, Box 218, Level 5, Cambridge Biomedical Campus, Cambridge CB2 0QQ, UK

2 Department of Radiology, Addenbrookes Hospital, Cambridge University Hospitals NHS Foundation Trust, Cambridge, UK

3 Breast screening Unit, Seacroft Hospital, York Road, Leeds LS146UH, UK extremely dense breasts when compared to $86 \%$ in fatty breasts, leading to 6 times more interval cancers [2]. This is partially due to the "masking effect" caused by the overlapping fibroglandular tissue, resulting in delayed diagnosis, and larger cancers with often poorer prognosis [3]. Breast density also contributes to increased risk of breast cancer with a 4.6fold increased risk for the densest group compared to the lowest density, fatty breasts[4].

Breast density can be measured using numerous methods and from images obtained on different modalities [5, 6]. Commonly, breast density is measured on digital mammography. Given its clinical importance, reader assigned (BIRADS) and automated breast density measures (area and volume) have been included in the latest risk prediction models. The best measure of density to determine risk, when used alone or in combination with other risk models (TyrerCuzick, BOADICEA, and Gail models), is yet to be determined [7-9]. Assessing density is important to target supplemental screening approaches for women with dense breasts. 
Various approaches and thresholds have been considered to guide the implementation of supplemental imaging, with studies evaluating which modality is best for this purpose $[10,11]$. The BRAID multicenter UK trial is comparing automated breast ultrasound (ABUS), abbreviated magnetic resonance imaging (ABB MRI), and contrast-enhanced spectral mammography (CESM) as a supplemental screening tool [12].

Many studies support the use of the whole breast ultrasound as an appropriate supplemental screening tool in dense breasts, due to its accessibility, lack of intravenous (IV) contrast and ionizing radiation, and better patient tolerance [13-18]. Handheld ultrasound (HHUS) increases the cancer detection rate by $1.8-4.6$ cancers per 1000 women screened, compared with mammography alone $[14,16,19,20]$. However, the use of HHUS as a screening tool has its own disadvantages, i.e., radiologist time required to perform the examination, significant operator dependence, higher recall rates, and relatively low positive predictive value. Unlike handheld ultrasound (HHUS), automated breast ultrasound (ABUS) is a three-dimensional volume imaging technique, offering proper orientation and documentation, leading to better reproducibility and making it easier to compare with subsequent follow-up studies. It can be performed independently and in a more standardized way with trained radiographers, saving radiologist time and improving efficiency [21].

\section{Technical Aspects}

The use of ABUS for breast cancer screening was introduced in 1980 [13], to deal with the shortcomings of mammography [22] in detecting cancer in women with dense breasts.

ABUS systems are available in two categories: supine [23, $24]$ and prone $[25,26]$. The latest supine $3 \mathrm{D}$ ABUS devices

\section{- Six standard volume acquisitions}

\section{RIGHT}
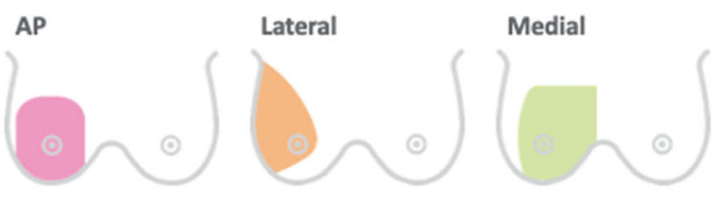

LEFT

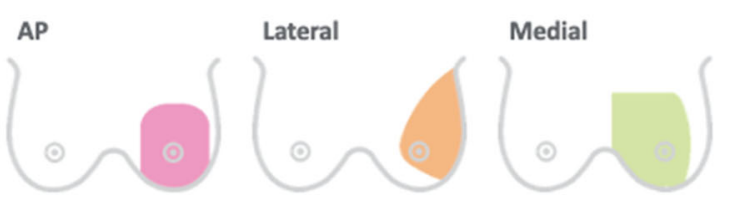

Fig 1 Overview of the ABUS scanning views with 3 standard views for each breast and 6 optional views based on patient body habitus, image used with

kind permission from GE Healthcare consist of an articulated arm with a long transducer, a touchscreen monitor, and a dedicated workstation for image interpretation. The transducers are automatically adjusted over their frequency range $(\sim 5-15 \mathrm{MHz})$, according to the chosen depth, producing $3 \mathrm{D}$ datasets with hundreds of images per acquisition at slice intervals of $\sim 2 \mathrm{~mm}$ without overlap. Technical advances of 3D ABUS systems consist in special software that enables optimized quality images with high resolution and uniformity throughout the image due to advanced reconstruction algorithms and automated adjustment of settings such as frequency, time gain compensation, harmonics, nipple shadow, and speckle reduction imaging [22].

A standard ABUS protocol consists of scanning each breast separately in three planes, anterior-posterior (AP), lateral (LAT), and medial (MED) positions, resulting in six images for both breasts. Additional views may be required in women with larger breasts to cover the whole area with four images (two per each breast) sufficient in smaller breasts (Fig. 1). Adequate patient positioning, transducer placement, proper depth selection, and compression are crucial in acquiring high-quality images with the total examination, including patient preparation, lasting approximately 15-20 min. Regular quality assurance tests should be performed as for ultrasound equipment. The volume data is processed automatically in multiplanar reconstruction (coronal and sagittal planes) and is transferred to a dedicated workstation for interpretation as full, reconstructed image sets cannot yet be viewed on picture archiving and communication system (PACS) workstations as standard.

\section{Artifacts and Pitfalls}

In a retrospective analysis of 1890 ABUS studies, the most common reasons for false-negative readings on ABUS exams

\section{Twelve optional volume acquisitions}

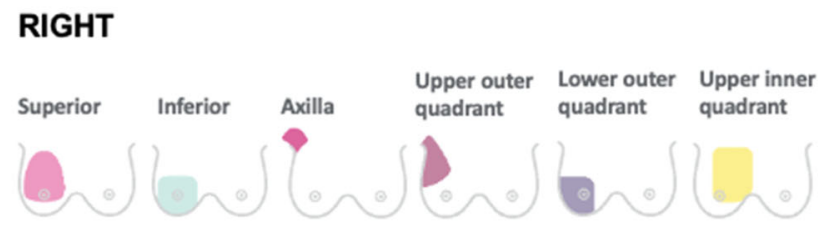

\section{LEFT}

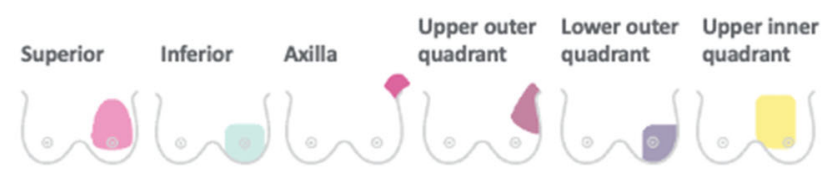


were reported as poor visibility, peripheral lesion location, and shadowing obscuring the lesion [27].

Technical artifacts such as loss-of-contact, dropout artifacts due to poor scanning or skin folding are the most common cause of poor visibility. Adequate patient positioning, uniform amount of lotion, and appropriate transducer placement and compression should be ensured to avoid this type of artifacts, while documenting skin lesions such as moles and surgical scars is also recommended (Fig. 2a). Nipple shadowing is another artifact, more commonly seen in inverted nipples; however, applying an abundance of waterbased lotion in this area as well as integrated software algorithms, such as tissue equalization algorithm and nipple shadow compensation, helps in reducing this type of artifact [21]. Hyperventilation, tachycardia, coughing, and talking are the common physiological conditions-related artifacts. Their repetitive sinusoidal wave pattern makes them easy to detect $[21,28]$; they are more prominent near the chest wall and can distort the deeper regions of the coronal and sagittal reformations. Breast lesion-related artifacts such as skip artifact and white wall sign are unique to ABUS and can assist in diagnosis/differentiation of specific lesions [28]. A skip artifact is caused when the transducer encounters a firm mass and presents as an artifactual horizontal line on the coronal plane (Fig. 2b); these may also occur due to prominent ribs. White wall signs are seen as hypoechoic circumscribed areas before a cyst in the coronal plane and are equivalent to the posterior enhancement in HHUS (Fig. 2c); their appearance is a typical sign of benign lesions [21].
Shadowing artifacts: This type of artifact is one of the major challenges in ABUS interpretation. It is crucial to distinguish between shadowing originating from normal breast parenchyma and those from true lesions due to their high correlation with malignant masses. "Wandering shadows" caused by the interference of ultrasound waves with curved surfaces of Cooper ligaments, presenting as repetitive linear shadows in the transverse plane (Fig. 2d), are common in ABUS examinations [21]. They tend to be more prominent in heterogeneous dense breasts, on the lateral and medial planes, and the periphery, potentially leading to misinterpretation [27]. Uniform compression and proper positioning are essential in reducing this type of artifact [22]. In a recent study, shadowing was classified in four categories, and a methodical approach was suggested to minimize false positives [28]. The persistence of an apparent lesion in more than one plane (axial and coronal/sagittal) is a strong positive finding that needs to be recalled. Sagittal plane might be helpful in certain cases to rule out true lesions (Fig. 2d).

\section{ABUS Implementations in a Screening Setting}

Many studies indicate that ABUS is a good supplemental screening tool for women with dense breasts, with a diagnostic performance similar to screening HHUS [29] and an increase of cancer detection rate by 1.9-7.7 cases per 1000 women compared to mammography alone [23, 30-33]. Sensitivity increased with $21.6-41.0 \%$, but specificity varied.
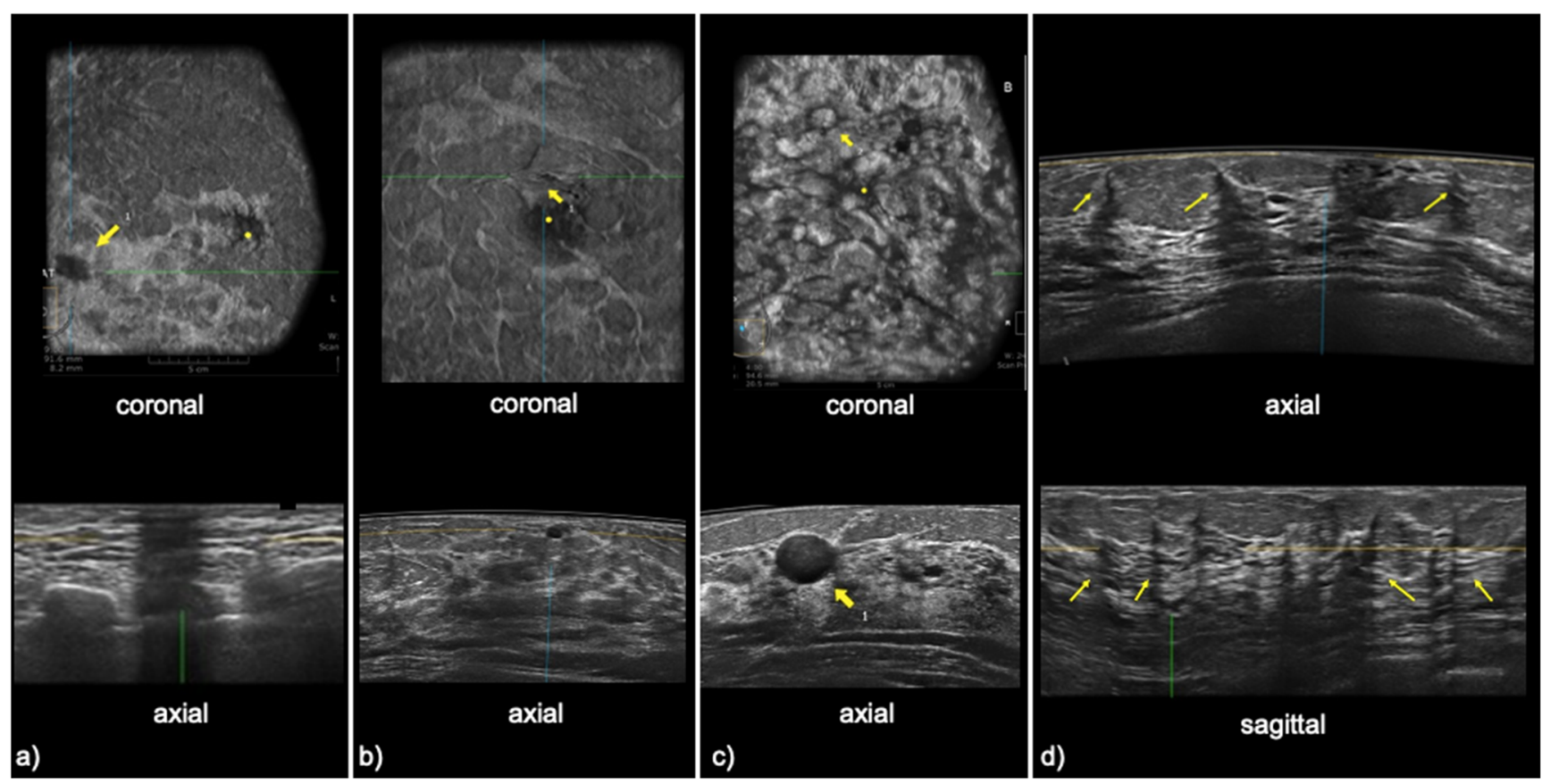

Fig. 2 Artifacts from ABUS (a) Contact artifact from a mole on both the coronal and saggital plane, shown by arrows. (b) Skip artifact from cyst on both the coronal and axial images. (c) White wall sign ; round echogenic area in coronal images, corresponding to the posterior enhancement of cyst in axial plane, shown by arrows (d) Wandering Shadows ; linear repetitive shadows in axial and sagittal planes, shown by arrows 
In the largest ABUS study (SomoInsight Study) of 15,318 women with dense breasts, the cancer detection rate was reported as 1.9 cases per 1000 women [23], similar to the results of Japan Strategic Anti-cancer Randomized Trial (J-START) [18] but lower than the results of American College of Radiology Imaging Network 6666 [16]; this difference was due to the different inclusion criteria of these studies. Most of the cancers detected were small, invasive, and predominantly node negative (Table 1).

Recall and biopsy rates tend to increase with ABUS as well (Table 1). These values have improved recently, due to increased reader experience and software developments with the latest ABUS systems $[35,36]$. This new modality has a learning curve, so adequate training and state-of-the-art examinations along with integration of computer-aided detection (CAD) software will potentially improve accuracy and reduce recall rates [37].

Recent European Society of Breast Imaging (EUSOBI) guidelines recommend the usage of HHUS or $3 \mathrm{D}$ ABUS as a supplemental screening modality following a negative mammogram in women of average or intermediate risk with dense breasts, $\mathrm{C}$ and $\mathrm{D}$, according to Breast Imaging Reporting and Data System (BI-RADS) Atlas, 5th edition [38, 39]. In addition, screening ABUS can be offered as an alternative to MRI in high-risk women in limited facilities or when MRI is contraindicated [11]. Incremental cancer detection rates are higher, between 2 and 3.6 per 1000 screens, for ABUS in comparison to digital breast tomosynthesis (DBT) (1-2 per 1000 screens) when used as a supplemental screening tool in women with dense breasts. These values are similar to HHUS but significantly lower than MRI and CESM. However, this comes at the expense of IV contrast administration, ionizing radiation, and higher recall rates [29]. In addition, MRI is not as widely accessible and requires more specialized training. Large multicentric randomized trials with long-term followup are needed to assess the feasibility of ABUS as an additional screening tool in reducing mortality rates [22].

\section{Computer-Aided Detection/Diagnosis}

A drawback of ABUS is the relatively long interpretation time due to a larger volume of images to be analyzed in both coronal and axial planes. The reading time reported so far varies significantly (2.9-9 $\mathrm{min}$ ), attributable to variation in radiologists' experience and complexity of cases [23, 29, 31, 40]. A CAD software for 3D ABUS (QVCAD ${ }^{\mathrm{TM}}$, QView Medical) has been recently developed and FDA approved [41]. Several studies have been investigating the benefits of concurrent-read CAD systems for interpretation of screening ABUS, and CAD seems to significantly reduce interpretation time, up to $35 \%$, as well as improving diagnostic accuracy [42-44]. Another approach to speed up reading is the use of coronal plane only [45], but more work on this is required to ensure that sensitivity does not drop. Further work is required to evaluate the feasibility of CAD in a screening setting.

In the ASSURE project, 120 unilateral ABUS exams chosen randomly were analyzed by eight experienced breast radiologists using CAD software. The reading time was significantly reduced by $15 \%$ overall [46]. CAD discarded $42.6 \%$ of BI-RADS $\geq 3$ lesions, $85.5 \%$ of which were benign, suggesting that CAD software could improve accuracy and potentially reduce unnecessary recalls [47].

\section{ABUS Implementations in Clinical Setting}

\section{Symptomatic Breast Imaging}

ABUS use in clinical practice is still under investigation. Several studies have reported similar diagnostic performance of ABUS to HHUS in differentiation of benign and malignant lesions (Table 2). In a recent meta-analysis of nine studies involving 1527 lesions, ABUS was found to have higher pooled sensitivity $(93 \%)$ and specificity $(86 \%)$ in detecting lesions,

Table 1 Results from 4 major studies examining supplemental screening with ultrasound [33, 34]

\begin{tabular}{|c|c|c|c|c|}
\hline Study & $\begin{array}{l}\text { SomoInsight } \\
\text { (Brem 2015) [23] }\end{array}$ & Wilczek (2016) [31] & $\begin{array}{l}\text { ACRIN 6666 } \\
\text { (Berg 2012) [16] }\end{array}$ & $\begin{array}{l}\text { J- START } \\
\text { (Ohuchi 2016) [18] }\end{array}$ \\
\hline Modality & ABUS & ABUS & HHUS & HHUS \\
\hline Study population & $\begin{array}{l}\text { Asymptomatic } \\
\text { women with dense } \\
\text { breasts }(15,318)\end{array}$ & $\begin{array}{l}\text { Asymptomatic women } \\
\text { with dense breasts (1668) }\end{array}$ & $\begin{array}{l}\text { Asymptomatic women } \\
\text { at high risk (2809) }\end{array}$ & $\begin{array}{l}\text { Asymptomatic women } \\
\text { in their } 40 \mathrm{~s}(36,752)\end{array}$ \\
\hline Cancer detection (rate per 1000) & 1.9 & 2.4 & 5.3 & 1.8 \\
\hline Net added recalls per screen $(\%)$ & 13.5 & 0.9 & 7.4 & 5.4 \\
\hline Invasive cancers $(\%)$ & 93.3 & NR & 93.7 & 82.0 \\
\hline Mean size of cancers (mm) & 12.9 & 21.8 & 10.0 & 14.2 \\
\hline Node negative cancers (\%) & 92.6 & 50.0 & 96.7 & 85.5 \\
\hline
\end{tabular}


Table 2 Major studies comparing sensitivity and specificity of ABUS (automatic breast ultrasound) and HHUS (handheld ultrasound) in clinical practice $[48,49]$

\begin{tabular}{|c|c|c|c|c|c|}
\hline \multirow[t]{2}{*}{ Study } & \multirow[t]{2}{*}{ Patients number } & \multicolumn{2}{|c|}{ Sensitivity $\%$} & \multicolumn{2}{|c|}{ Specificity $\%$} \\
\hline & & ABUS & US & ABUS & US \\
\hline Cho et al. [50] & 141 & 98.3 & 96.7 & 96.7 & 64.4 \\
\hline Kotsianos-Hermle et al. [51] & 97 & 95 & 97 & 93 & 88 \\
\hline Wang HY et al. [52] & 213 & 95.3 & 90.6 & 80.5 & 82.5 \\
\hline Wang ZL et al. [53] & 155 & 96.1 & 93.2 & 91.9 & 88,7 \\
\hline Chen et al. [54] & 175 & 92.5 & 88.1 & 86.2 & 87.5 \\
\hline Jeh et al. [55] & 173 & 88 & 95.7 & 76.2 & 49.4 \\
\hline Niu et al. [56] & 398 & 92.23 & 82.52 & 77.62 & 80.24 \\
\hline
\end{tabular}

compared to HHUS ( $90 \%$ and $82 \%$, respectively) [48]. However, these are small studies with relatively low number of lesions. Looking at interobserver reliability in BI-RADS assessment, heterogeneous results with considerable variation in kappa values have been reported [57]. Vourtsis and Katchulis found a very high (99.8 $\%$; kappa $=0.994, \mathrm{p}<0.0001)$ interobserver agreement in BI-RADS classification between 3D ABUS and HHUS, in a retrospective study of 1886 women, with ABUS superiority in detecting architectural distortion [29]. Another recently published multicentric study of 937 Chinese women with dense breasts, evaluating the diagnostic performance of ABUS and HHUS in combination with mammography, reported significant improvement in cancer detection rate as well as strong correlation between ABUS and HHUS, with an accuracy of $93 \%$ for ABUS and $92 \%$ for HHUS and $94 \%$ agreement between them [58].

The coronal plane is a specific feature of ABUS that enables the visualization of a lesion in the anatomical plane and contributes to better detection and characterization $[59,60]$. One of the unique findings of coronal plane is the so-called retraction phenomenon, frequently a sign of architectural distortion and malignancy (Fig. 3) that can supplement mammography in detecting noncalcified carcinomas in women with dense breasts [29]. Zheng et al. reported that the retraction sign and microlobulated margins have both high diagnostic values in distinguishing between benign and malignant breast masses in ABUS [60].

A recent study analyzing 457 lesions in coronal plane, including 80 non-mass lesions, concluded that the retraction phenomenon is highly predictive of malignancy; continuous hyper- or hypoechoic rims were more associated with benign lesions, whereas discontinuous rims were suggestive of malignancies. For the nonmass lesions, the skip artifact was found to correlate more with malignant lesions [61]. In addition, ABUS seems to outperform HHUS in volumetric measurements of lesions, with significant higher accuracy [62, 63].

Local Staging Breast magnetic resonance imaging (MRI) is the gold standard in the evaluation of disease extent and treatment planning. 3D ABUS could be a good alternative to MRI in finding additional lesions due to its three-dimensional orientation and repeatability (Fig. 3). In a recent study comparing the diagnostic value of ABUS to HHUS with MRI as gold standard, they reported similar diagnostic accuracy for ABUS and HHUS (87.2\% and $89.7 \%$ ) but better size correlation between ABUS and MRI $(r=0.89)$ compared to HHUS $(r=$ 0.82) [64]. In a similar study including 100 index cancers, ABUS showed better agreement with histology than HHUS with a higher intraclass correlation (ICC), 0.85 vs 0.75 , respectively [65]. Furthermore, the coronal plane provides better segmental approach, and global visualization of the tumor with similar surgical orientation therefore might be useful in surgical planning (Fig. 4). In another analysis of 142 biopsy-proven DCIS cases examining the use of ABUS in guiding breast conservation surgery, ABUS was superior to HHUS in both surgery planning and predicting recurrence, with a detection rate significantly higher $\left(\chi^{2}=268.000, \mathrm{P}<\right.$ 0.001) [63]. Semiautomated and automated algorithms for ABUS lesion segmentation and volume measuring are under investigation [66,67].

Monitoring Response to Neoadjuvant Chemotherapy US can be a potentially useful imaging modality for early prediction of pathological response to NAC [68]. ABUS is a promising tool, offering better orientation and reproducibility in comparison to HHUS. In a recent study, Wang et al. reported high sensitivity $(88.1 \%)$ and specificity $(81.5 \%)$ of ABUS coronal plane, in predicting pathological complete response (pCR) rate after four cycles of chemotherapy [69]. They concluded that 
Fig. 3 A multifical grade II invasive carcinoma of no special type (NST) with intratumoral low grade DCIS, presenting with (a) "The retraction sign" (circle) and two foci of tumour, $18 \mathrm{~mm}$ and 6 mm (arrows) on ABUS coronal plane and (b) corresponding HHUS images

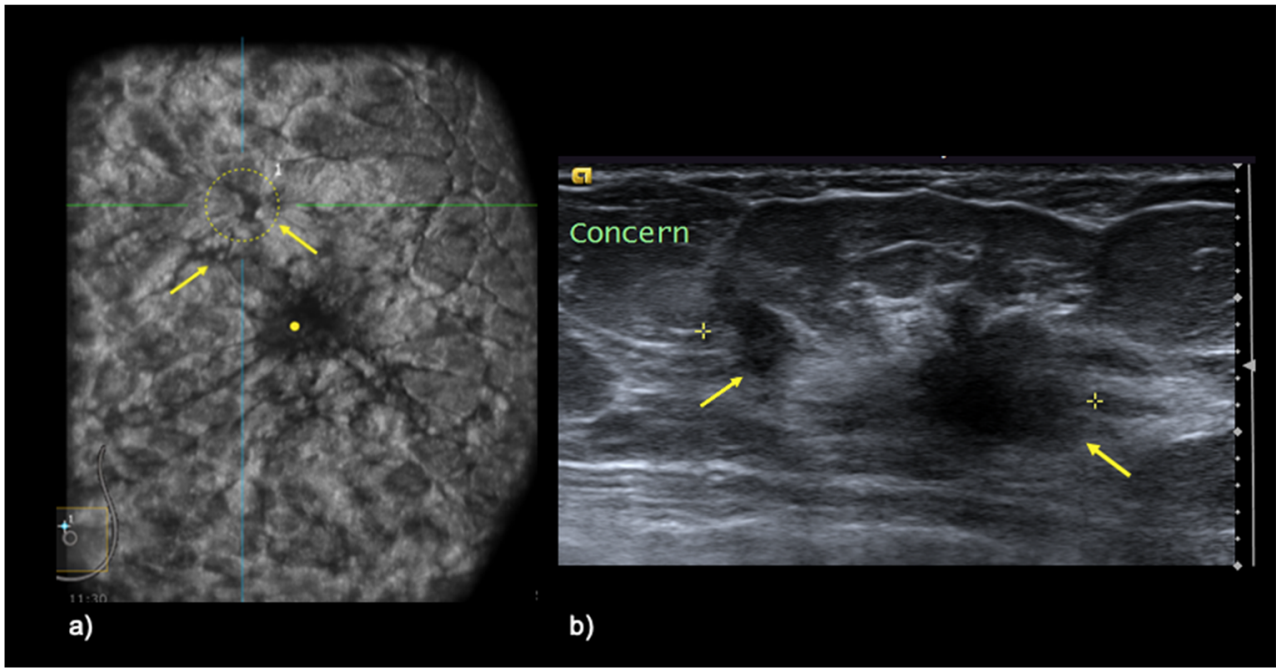

ABUS is a useful tool in early evaluation of pathological complete response after 2 cycles of NAC while less reliable when predicting poor pathological outcomes.

Second-look ABUS Another potential application of ABUS is the further evaluation of MRI findings instead of second-look HHUS. Recent studies have shown that ABUS is better than
HHUS and with similar performance to MRI in predicting breast cancer size and finding additional lesions [65, 70, 71]. Therefore, it can be a good alternative to MRI in guiding breast conservation surgery and predicting recurrence [63].

However, there are some limitations of ABUS such as lack of image-guided biopsy and axilla and tumor vascularity assessment.
Fig. 4 Irregular $9 \mathrm{~mm}$ hypoechoic lesion on ABUS (a) coronal and (b) axial planes (circle) , corresponding (c) postcontrast MRI and (d) secondlook US images (arrows). Unifocal Invasive Lobular Carcinoma with very good correlation of lesion size and location between MRI and ABUS

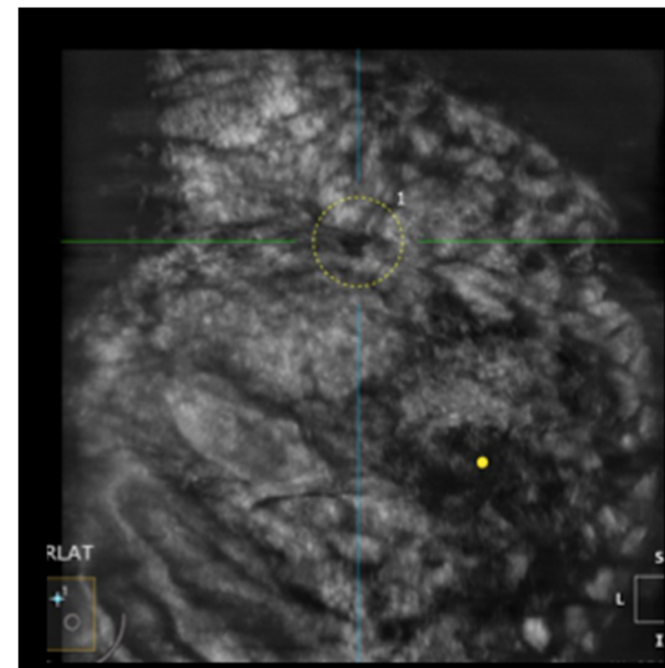

a)

c)
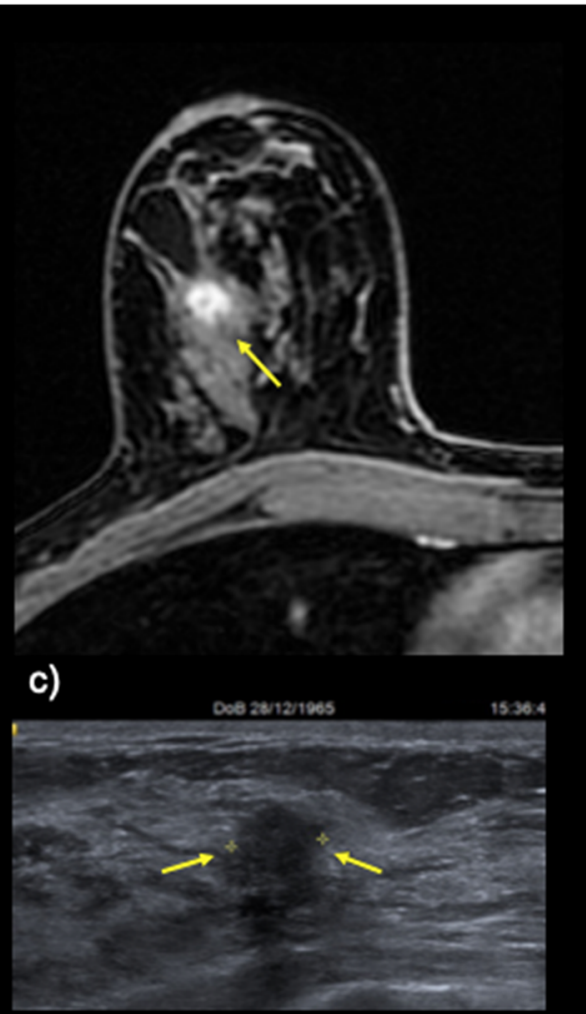

d) 


\section{Future Perspectives}

Ongoing research is looking at correlation between molecular subtypes of breast cancer and ABUS morphological features. In a recent analysis of 303 malignant lesions, strong correlation was found between their specific imaging features on ABUS such as retraction, post-acoustic shadowing, echogenic halo, calcifications, and molecular subtypes, especially for the "retraction phenomenon" on coronal views, as the strongest independent predictor for the luminal-A subtype when present and for the triple-negative subtype when absent [72].

Other possible implementations of ABUS include breast density evaluation $[54,73]$. In their study, Chen et al. found a high correlation of density results between MRI and ABUS, for whole breast volume $(\mathrm{r}=0.798)$ and for breast percentage density $(r=0.825)$ [74].

ABUS can be used for follow-up of benign lesions, due to its precise documentation and orientation. In a retrospective study evaluating the reproducibility of ABUS, readers ICCs for lesion location (clock face location, distance from nipple) and size were reported as $0.994,0.926$, and 0.980 , respectively, suggesting high reliability [75].

Fusion-X-US is a new device under investigation, combining ABUS and tomosynthesis in one device, aiming to improve workflow in clinical practice. Preliminary results of a prospective study, analyzing 101 patients with this prototype, showed good breast coverage $(80.0 \%)$ and diagnostic accuracy of ABUS (85.0\%), as well as $97.1 \%$ lesion identification with the combined system [76].

\section{Conclusions}

ABUS is a good supplemental screening tool for women with dense breasts and should be considered as an alternative to other modalities due to good patient tolerance, lack of ionizing radiation, and IV contrast. CAD is a promising tool in reducing interpretation time and improving ABUS accuracy. Other possible applications include use in symptomatic clinics in younger women and for surveillance of benign lesions, local staging, monitoring response to NAC, second-look tool, correlation with molecular subtypes of breast cancer, and breast density evaluation. Further developments are expected in the field of deep learning and integration of radiomics. Larger studies, robust training, and software incorporation and standardization are required for better implementation of this imaging modality in screening and diagnostic setting.

Acknowledgements This work is supported by the NIHR Cambridge Biomedical Research Centre. Funding and research support is received from Cancer Research UK, GE Healthcare, and Bayer.

Availability of Data and Materials Not applicable
Code Availability Not applicable

Competing Interests I Allajbeu: no conflict of interest to disclose S Hickman: has research collaborations with Merantix, ScreenPoint, Volpara, and Lunit

N Payne: GE Healthcare funding for research

P L Moyle: no conflict of interest to disclose

K Taylor: no conflict of interest to disclose

N Sharma: GE Healthcare funding for research

F J Gilbert: GE Healthcare funding for research

Author Contribution All authors have made substantial contribution to the design of this article, review and interpretation of the relevant literature, as well as providing critical feedback according to their field of expertise.

Funding This work was supported by the CRUK grant (C543/A26884).

\section{Declarations}

Ethics Approval Not applicable

Consent to Particip0061te Not applicable

Consent for Publication Yes

Open Access This article is licensed under a Creative Commons Attribution 4.0 International License, which permits use, sharing, adaptation, distribution and reproduction in any medium or format, as long as you give appropriate credit to the original author(s) and the source, provide a link to the Creative Commons licence, and indicate if changes were made. The images or other third party material in this article are included in the article's Creative Commons licence, unless indicated otherwise in a credit line to the material. If material is not included in the article's Creative Commons licence and your intended use is not permitted by statutory regulation or exceeds the permitted use, you will need to obtain permission directly from the copyright holder. To view a copy of this licence, visit http://creativecommons.org/licenses/by/4.0/.

\section{References}

1. Marmot M, Altman DG, Cameron DA, Dewar JA, Thompson SG, Wilcox M. The benefits and harms of breast cancer screening: an independent review. Lancet. 2012;380:1778-86. https://doi.org/10. 1016/S0140-6736(12)61611-0.

2. Wanders JOP, Holland K, Veldhuis WB, Mann RM, Pijnappel RM, Peeters PHM, et al. Volumetric breast density affects performance of digital screening mammography. Breast Cancer Res Treat Springer US. 2017;162:95-103.

3. Bae MS, Moon WK, Chang JM, Koo HR, Kim WH, Cho N, et al. Breast cancer detected with screening US: reasons for nondetection at mammography. Radiology. 2014;270:369-77.

4. McCormack VA, Dos Santos Silva I. Breast density and parenchymal patterns as markers of breast cancer risk: a meta-analysis. Cancer Epidemiol Biomark Prev. 2006;15:1159-69.

5. Vinnicombe SJ. Breast density: why all the fuss? Clin Radiol. 2018;73:334-57. https://doi.org/10.1016/j.crad.2017.11.018.

6. Lian J, Li K. A review of breast density implications and breast cancer screening. Clin Breast Cancer. 2020;S1526-8209:30060-4.

7. Astley SM, Harkness EF, Sergeant JC, Warwick J, Stavrinos P, Warren R, et al. A comparison of five methods of measuring 
mammographic density: a case-control study. Breast Cancer Res Breast Cancer Research. 2018;20:1-13.

8. Brentnall AR, Harkness EF, Astley SM, Donnelly LS, Stavrinos P, Sampson S, et al. Mammographic density adds accuracy to both the Tyrer-Cuzick and Gail breast cancer risk models in a prospective UK screening cohort. Breast Cancer Res Breast Cancer Research. 2015;17:1-10.

9. Terry MB, Liao Y, Whittemore AS, Leoce N, Buchsbaum R, Zeinomar N, et al. 10-year performance of four models of breast cancer risk: a validation study. Lancet Oncol. 2019;20:504-17. https://doi.org/10.1016/S1470-2045(18)30902-1.

10. Clinical Trials.gov. Breast screening - risk adaptive imaging for density (BRAID) [Internet]. 2019 [cited 2020 Jun 15]. Available from: https://clinicaltrials.gov/ct2/show/NCT04097366

11. Kerlikowske K, Sprague BL, Tosteson ANA, Wernli KJ, Rauscher $\mathrm{GH}$, Johnson D, et al. Strategies to identify women at high risk of advanced breast cancer during routine screening for discussion of supplemental imaging. JAMA Intern Med. 2019;179:1230-9.

12. ClinicalTrials.gov. Breast screening - risk adaptive imaging for density (BRAID) [Internet]. [cited 2020 Oct 27]. Available from: https://clinicaltrials.gov/ct2/show/NCT04097366

13. Kolb TM, Lichy J, Newhouse JH. Comparison of the performance of screening mammography, physical examination, and breast US and evaluation of factors that influence them: an analysis of 27,825 patient evaluations. Radiology. 2002;225:165-75.

14. Berg WA, Blume JD, Cormack JB, Mendelson EB, Lehrer D, Böhm-Vélez M, et al. Combined screening with ultrasound and mammography vs mammography alone in women at elevated risk of breast cancer. JAMA - J Am Med Assoc. 2008;299:2151-63.

15. Corsetti V, Houssami N, Ferrari A, Ghirardi M, Bellarosa S, Angelini O, et al. Breast screening with ultrasound in women with mammography-negative dense breasts: evidence on incremental cancer detection and false positives, and associated cost. Eur J Cancer. 2008;44:539-44.

16. Berg WA, Zhang Z, Lehrer D, Jong RA, Pisano ED, Barr RG, et al. Detection of breast cancer with addition of annual screening ultrasound or a single screening MRI to mammography in women with elevated breast cancer risk. JAMA - J Am Med Assoc. 2012;307:1394- 404.

17. Hooley RJ, Greenberg KL, Stackhouse RM, Geisel JL, Butler RS, Philpotts LE. Screening US in patients with mammographically dense breasts: Initial experience with Connecticut public act 0941. Radiology. 2012;265:59-69.

18. Ohuchi N, Suzuki A, Sobue T, Kawai M, Yamamoto S, Zheng YF, et al. Sensitivity and specificity of mammography and adjunctive ultrasonography to screen for breast cancer in the Japan Strategic Anti-cancer Randomized Trial (J-START): a randomised controlled trial. Lancet. 2016;387:341-8. https://doi.org/10.1016/ S0140-6736(15)00774-6.

19. Corsetti V, Houssami N, Ghirardi M, Ferrari A, Speziani M, Bellarosa S, et al. Evidence of the effect of adjunct ultrasound screening in women with mammography-negative dense breasts: interval breast cancers at 1 year follow-up. Eur J Cancer. 2011;47: 1021-6. https://doi.org/10.1016/j.ejca.2010.12.002.

20. Sprague BL, Stout NK, Schechter C, Van Ravesteyn NT, Cevik M, Alagoz O, et al. Benefits, harms, and cost-effectiveness of supplemental ultrasonography screening for women with dense breasts. Ann Intern Med. 2015;162:157-66.

21. van Zelst JCM, Mann RM. Automated three-dimensional breast US for screening: technique, artifacts, and lesion characterization. Radiographics. 2018;38:663-83.

22. Vourtsis A. Three-dimensional automated breast ultrasound: technical aspects and first results. Diagn Interv Imaging. 2019;100:579-92.

23. Brem RF, Tabár L, Duffy SW, Inciardi MF, Guingrich JA, Hashimoto $\mathrm{BE}$, et al. Assessing improvement in detection of breast cancer with threedimensional automated breast US in women with dense breast tissue: the SomoInsight study. Radiology. 2015;274:663-73.
24. Mundinger A. 3D supine automated ultrasound (SAUS, ABUS, ABVS) for supplemental screening women with dense breasts. J Breast Heal. 2016;12:52-5.

25. Farrokh A, Erdönmez H, Schäfer F, Maass N. SOFIA: a novel automated breast ultrasound system used on patients in the prone position: a pilot study on lesion detection in comparison to handheld grayscale ultrasound. Geburtshilfe Frauenheilkd. 2018;78:499-505.

26. O'Flynn EAM, Fromageau J, Ledger AE, Messa A, D'Aquino A, Schoemaker MJ, et al. Ultrasound tomography evaluation of breast density: a comparison with noncontrast magnetic resonance imaging. Investig Radiol. 2017;52:343-8.

27. Grubstein A, Rapson Y, Gadiel I, Cohen M. Analysis of falsenegative readings of automated breast ultrasound studies. J Clin Ultrasound. 2017;45:245-51.

28. Karst I, Henley C, Gottschalk N, Floyd S, Mendelson EB. Threedimensional automated breast US: facts and artifacts. Radiographics. 2019;39:913-31.

29. Vourtsis A, Kachulis A. The performance of $3 \mathrm{D}$ ABUS versus HHUS in the visualisation and BI-RADS characterisation of breast lesions in a large cohort of 1,886 women. Eur Radiol European Radiology. 2018;28:592-601.

30. Kelly KM, Dean J, Comulada WS, Lee SJ. Breast cancer detection using automated whole breast ultrasound and mammography in radiographically dense breasts. Eur Radiol. 2010;20:734 42.

31. Wilczek B, Wilczek HE, Rasouliyan L, Leifland K. Adding 3D automated breast ultrasound to mammography screening in women with heterogeneously and extremely dense breasts: report from a hospital-based, high-volume, single-center breast cancer screening program. Eur J Radiol. 2016;85:1554-63. https://doi.org/10.1016/j. ejrad.2016.06.004.

32. Giuliano V, Giuliano C. Improved breast cancer detection in asymptomatic women using 3D-automated breast ultrasound in mammographically dense breasts. Clin Imaging. 2013;37:480-6. https://doi.org/10.1016/j.clinimag.2012.09.018.

33. Kim SH, Kim HH, Moon WK. Automated breast ultrasound screening for dense breasts. Korean J Radiol. 2020;21:15-24.

34. Vourtsis A, Berg WA. Breast density implications and supplemental screening. Eur Radiol. 2019;29:1762-77.

35. Berg WA, Vourtsis A. Screening breast ultrasound using handheld or automated technique in women with dense breasts. J Breast Imaging. 2019;1:283-96.

36. Arleo EK, Saleh M, Ionescu D, Drotman M, Min RJ, Hentel K. Recall rate of screening ultrasound with automated breast volumetric scanning (ABVS) in women with dense breasts: a first quarter experience. Clin Imaging. 2014;38:439-44. https://doi.org/10. 1016/j.clinimag.2014.03.012.

37. Mendelson EB, Berg WA. Training and standards for performance, interpretation, and structured reporting for supplemental breast cancer screening. Am J Roentgenol. 2015;204:265-8.

38. Evans A, Trimboli RM, Athanasiou A, Balleyguier C, Baltzer PA, Bick U, et al. Breast ultrasound: recommendations for information to women and referring physicians by the European Society of Breast Imaging. Insights Imaging. 2018;9:449-61.

39. D'Orsi CJ, Sickles EA, Mendelson EB, et al. ACR BI-RADS® Atlas, Breast Imaging Reporting and Data System. Reston, VA: American College of Radiology; 2013.

40. Huppe AI, Inciardi MF, Redick M, Carroll M, Buckley J, Hill JD, et al. Automated breast ultrasound interpretation times: a reader performance study. Acad Radiol. 2018;25:1577-81.

41. USA Food \& Drug Administration. Premarket approval (PMA) [Internet]. [cited 2020 Oct 27]. Available from: https://www. accessdata.fda.gov $/ \mathrm{scripts} / \mathrm{cdrh} / \mathrm{cfdocs} / \mathrm{cfpma} / \mathrm{pma} . \mathrm{cfm}$ ? id= P150043

42. Jiang Y, Inciardi MF, Edwards AV, Papaioannou J, et al. AJR Am J Roentgenol. 2018:452-61. 
43. Yang S, Gao X, Liu L, Shu R, Yan J, Zhang G, et al. Performance and reading time of automated breast us with or without computeraided detection. Radiology. 2019;292:540-9.

44. van Zelst JCM, Tan T, Clauser P, Domingo A, Dorrius MD, Drieling D, et al. Dedicated computer-aided detection software for automated 3D breast ultrasound; an efficient tool for the radiologist in supplemental screening of women with dense breasts. Eur Radiol European Radiology. 2018;28:2996-3006.

45. Schiaffino S, Gristina L, Tosto S, Massone E, De Giorgis S, Garlaschi A, et al. The value of coronal view as a stand-alone assessment in women undergoing automated breast ultrasound. Springer Milan: Radiol Medica; 2020.

46. European Commission - CORDIS EU Research Results. Adapting breast cancer screening strategy using personalised risk estimation [Internet]. 2016 [cited 2020 Dec 14]. Available from: https://cordis. europa.eu/project/id/306088/reporting

47. van Zelst JCM, Tan T, Mann RM, Karssemeijer N. Validation of radiologists' findings by computer-aided detection (CAD) software in breast cancer detection with automated 3D breast ultrasound: a concept study in implementation of artificial intelligence software. Acta Radiol. 2020;61:312-20.

48. Zhang X, Chen J, Zhou Y, Mao F, Lin Y, Shen S, et al. Diagnostic value of an automated breast volume scanner compared with a hand-held ultrasound: a meta-analysis. Gland Surg. 2019;8:698711.

49. Nicosia L, Ferrari F, Bozzini AC, Latronico A, Trentin C, Meneghetti L, et al. Automatic breast ultrasound: state of the art and future perspectives. Ecancermedicalscience. 2020;14:1-13.

50. Cho N, Moon WK, Cha JH, Kim SM, Han BK, Kim EK, et al. Differentiating benign from malignant solid breast masses: comparison of two-dimensional and three-dimensional US. Radiology. 2006;240:26-31.

51. Kotsianos-Hermle D, Hiltawsky KM, Wirth S, Fischer T, Friese K, Reiser M. Analysis of 107 breast lesions with automated 3D ultrasound and comparison with mammography and manual ultrasound. Eur J Radiol. 2009;71:109-15.

52. Wang HY, Jiang YX, Zhu QL, Zhang J, Dai Q, Liu H, et al. Differentiation of benign and malignant breast lesions: a comparison between automatically generated breast volume scans and handheld ultrasound examinations. Eur J Radiol. 2012;81:3190200.

53. Wang ZL, Xw JH, Li JL, Huang Y, Tang J. Comparison of automated breast volume scanning to hand-held ultrasound and mammography. Radiol Med. 2012;117:1287-93.

54. Chen L, Chen Y, Diao XH, Fang L, Pang Y, Cheng AQ, et al. Comparative study of automated breast 3-D ultrasound and handheld B-mode ultrasound for differentiation of benign and malignant breast masses. Ultrasound Med Biol. 2013;39:1735-42.

55. Jeh SK, Kim SH, Choi JJ, Jung SS, Choe BJ, Park S, et al. Comparison of automated breast ultrasonography to handheld ultrasonography in detecting and diagnosing breast lesions. Acta Radiol. 2016;57:162-9.

56. Niu L, Bao L, Zhu L, Tan Y, Xu X, Shan Y, et al. Diagnostic performance of automated breast ultrasound in differentiating benign and malignant breast masses in asymptomatic women: a comparison study with handheld ultrasound. J Ultrasound Med. 2019;38:2871-80.

57. Meng Z, Chen C, Zhu Y, Zhang S, Wei C, Hu B, et al. Diagnostic performance of the automated breast volume scanner: a systematic review of inter-rater reliability/agreement and meta-analysis of diagnostic accuracy for differentiating benign and malignant breast lesions. Eur Radiol. 2015;25:3638-47.

58. Jia M, Lin X, Zhou X, Yan H, Chen Y, Liu P, et al. Diagnostic performance of automated breast ultrasound and handheld ultrasound in women with dense breasts. Breast Cancer Res Treat Springer US. 2020;181:589-97.
59. Van Zelst JCM, Platel B, Karssemeijer N, Mann RM. Multiplanar reconstructions of $3 \mathrm{D}$ automated breast ultrasound improve lesion differentiation by radiologists. Acad Radiol. 2015;22:1489-96. https://doi.org/10.1016/j.acra.2015.08.006.

60. Zheng FY, Yan LX, Huang BJ, Xia HS, Wang X, Lu Q, et al. Comparison of retraction phenomenon and BI-RADS-US descriptors in differentiating benign and malignant breast masses using an automated breast volume scanner. Eur J Radiol. 2015;84:2123-9. https://doi.org/10.1016/j.ejrad.2015.07.028.

61. Tang G, An X, Xiang H, Liu L, Li A, Lin X. Automated breast ultrasound: interobserver agreement, diagnostic value, and associated clinical factors of coronal-plane image features. Korean $\mathrm{J}$ Radiol. 2020;21:550-60.

62. Li N, Jiang YX, Zhu QL, Zhang J, Dai Q, Liu H, et al. Accuracy of an automated breast volume ultrasound system for assessment of the Pre-operative extent of pure ductal carcinoma in situ: comparison with a conventional handheld ultrasound examination. Ultrasound Med Biol. 2013;39:2255-63.

63. Huang A, Zhu L, Tan Y, Liu J, Xiang J, Zhu Q, et al. Evaluation of automated breast volume scanner for breast conservation surgery in ductal carcinoma in situ. Oncol Lett. 2016;12:2481-4.

64. Schmachtenberg C, Fischer T, Hamm B, Bick U. Diagnostic performance of automated breast volume scanning (ABVS) compared to handheld ultrasonography with breast MRI as the gold standard. Acad Radiol. 2017;24:954-61.

65. Girometti R, Zanotel M, Londero V, Linda A, Lorenzon M, Zuiani C. Automated breast volume scanner (ABVS) in assessing breast cancer size: a comparison with conventional ultrasound and magnetic resonance imaging. Eur Radiol European Radiology. 2018;28: $1000-8$.

66. Lee CY, Chang TF, Chou YH, Yang KC. Fully automated lesion segmentation and visualization in automated whole breast ultrasound (ABUS) images. Quant Imaging Med Surg. 2020;10:56884.

67. Agarwal R, Diaz O, Lladó X, Gubern-Mérida A, Vilanova JC, Martí R. Lesion segmentation in automated 3D breast ultrasound: volumetric analysis. Ultrason Imaging. 2018;40:97-112. https:// doi.org/10.1177/0161734617737733.

68. Marinovich ML, Houssami N, Macaskill P, Von Minckwitz G, Blohmer JU, Irwig L. Accuracy of ultrasound for predicting pathologic response during neoadjuvant therapy for breast cancer. Int J Cancer. 2015;136:2730-7.

69. Wang X, Huo L, He Y, Fan Z, Wang T, Xie Y, et al. Early prediction of pathological outcomes to neoadjuvant chemotherapy in breast cancer patients using automated breast ultrasound. Chin J Cancer Res. 2016;28:478-85.

70. Kim Y, Kang BJ, Kim SH, Lee EJ. Prospective study comparing two second-look ultrasound techniques: handheld ultrasound and an automated breast volume scanner. J Ultrasound Med. 2016;35: 2103-12.

71. Girometti R, Zanotel M, Londero V, Bazzocchi M, Zuiani C. Comparison between automated breast volume scanner (ABVS) versus hand-held ultrasound as a second look procedure after magnetic resonance imaging. Eur Radiol European Radiology. 2017;27:3767-75.

72. Zheng FY, Lu Q, Huang BJ, Xia HS, Yan LX, Wang X, et al. Imaging features of automated breast volume scanner: correlation with molecular subtypes of breast cancer. Eur J Radiol. 2017. Available from:;86:267-75. https://doi.org/10.1016/j.ejrad.2016. 11.032.

73. Moon WK, Shen YW, Huang CS, Luo SC, Kuzucan A, Chen JH, et al. Comparative study of density analysis using automated whole breast ultrasound and MRI. Med Phys. 2011;38:382-9.

74. Chen JH, Lee YW, Chan SW, Yeh DC, Chang RF. Breast density analysis with automated whole-breast ultrasound: comparison with 
3-D magnetic resonance imaging. Ultrasound Med Biol. 2016;42: 1211-20.

75. Chang JM, Cha JH, Park JS, Kim SJ, Moon WK. Automated breast ultrasound system (ABUS): Reproducibility of mass localization, size measurement, and characterization on serial examinations. Acta Radiol. 2015;56:1163-70.

76. Schäfgen B, Juskic M, Radicke M, Hertel M, Barr R, Pfob A, et al. Evaluation of the FUSION-X-US-II prototype to combine automated breast ultrasound and tomosynthesis. Eur Radiol. 2020;31:3712-20. https://doi.org/10.1007/s00330-020-07573-3.

Publisher's Note Springer Nature remains neutral with regard to jurisdictional claims in published maps and institutional affiliations. 